\title{
Automated tracking of quantitative parameters from single line scanning of vocal folds: A case study of the 'messa di voce' exercise
}

\author{
PHILIPPE H. DEJONCKERE ${ }^{1}$, JEAN LEBACQ ${ }^{2}$, LEONARDO BOCCHI ${ }^{3}$, \\ SILVIA ORLANDI ${ }^{3} \&$ CLAUDIA MANFREDI ${ }^{3}$
}

${ }^{1}$ KULeuven University of Leuven, Neurosciences, Belgium; Federal Institute of Occupational Diseases, Av. de l'Astronomie, 1, B-1210 Brussels, Belgium, ${ }^{2}$ Institute of Neuroscience CEMO, Université Catholique de Louvain, Avenue Hippocrate 55 bte B1.55.12 B-1200 Brussels, Belgium, and ${ }^{3}$ Department of Information Engineering, Università degli Studi di Firenze, Via S. Marta 3, 50139 Firenze, Italy

\begin{abstract}
This article presents a novel application of the 'single line scanning' of the vocal fold vibrations (kymography) in singing pedagogy, particularly in a specific technical voice exercise: the 'messa di voce'. It aims at giving the singer relevant and valid short-term feedback. A user-friendly automatic analysis program makes possible a precise, immediate quantification of the essential physiological parameters characterizing the changes in glottal impedance, concomitant with the progressive increase and decrease of the lung pressure. The data provided by the program show a strong correlation with the hand-made measurements. Additional measurements such as subglottic pressure and flow glottography by inverse filtering can be meaningfully correlated with the data obtained from the kymographic images.
\end{abstract}

Key words: Feedback, flow glottography, high speed, messa di voce, singing, single line scanning, subglottic pressure, videokymography

\section{Introduction}

This article presents a novel and original application of the 'single line scanning' of the vocal fold vibrations (kymography) in singing pedagogy. Short-term feedback for relevant physiological parameters of voice production may be very useful for the singer, particularly in acquiring specific technical skills based on motor control, e.g. for producing a 'messa di voce'. The application actually consists of a detailed analysis of several biomechanical parameters over time during the singing exercise. Achieving this in quasireal time requires a valid and user-friendly automatic analysis program of the kymographic images, which appears indispensable for opening such new applications in the field of voice pedagogy.

\section{Single line scanning}

Single line scanning of vocal fold vibrations (kymography, or videokymography; VKG) (1) is an imaging method based on a special digital camera, which can operate in two different modes: standard and high-speed. The camera is fixed on a rigid $70^{\circ}$ or $90^{\circ}$ endoscope. In the standard mode, the camera provides standard images displaying all of the vocal folds at standard video frame rate $(30 / 25$ frames/s, with $720 \times 486 / 768 \times 576$ pixel resolution). In the high-speed mode, the video camera delivers images from a single line selected from the whole image, at the speed of approximately $7875 / 7812.5$ line-images/s and $720 \times 1 / 768 \times 1$ pixels resolution, depending on the video format. The selected line is usually at the level of the mid-portion of the vibrating folds. The resulting high-speed image, called a 'kymogram', displays the vibratory pattern of the small selected part of the vocal folds cycle by cycle. A kymographic recording is divided into video frames, i.e. segments of approx. 15-18 ms duration (time is on the vertical axis, up to down). 
When correctly applied, the technique allows a clear visualization of some essential physiological parameters of vocal fold vibration: period; duration of opening, closing, and closed phases; amplitude of the vibration; and right-left symmetry (Figure 1).

Kymography has been applied successfully to voice pathology (e.g. 2-5), particularly for situations where traditional videostroboscopy is failing, as in the case of very irregular vibrations, or when the vocal folds do not vibrate at the same frequency, or in cases of short 'accidents', e.g. register breaks.

Another potential field of application of the technique is singing voice pedagogy, as it provides realtime visual feedback for the subject and allows short-term inspection and analysis of the recorded sequence. This is particularly relevant for singing exercises requiring critical adjustments at vocal fold level that can be controlled and trained by the singer. Clinical experience teaches that many singers become quickly accustomed to the presence of the scope and remain able to achieve practically any pitch, loudness, register, arpeggio, etc.

The method becomes still more interesting with the input of automatic quantitative analysis of the above-mentioned parameters, as it has already been achieved for pathology, but focusing on asymmetries and irregularities $(2,5)$, which is a different scope. Actually the kymogram-contrary to the complex image of the whole endolarynx - can be adequately described by these few measures. Important is of course an adequate line selection, which is achieved by appropriately tilting the endoscope (6).

In this work, the potential application of automatic analysis of kymograms is demonstrated in the specific case of the 'messa di voce'. Because of the novelty of the application, and the focus on parameters that differ from those of interest in pathology, a necessary validation of the automatic analysis by comparison with manual measurements is achieved.

\section{'Messa di voce': its importance in singing pedagogy}

The 'messa di voce', which The New Harvard Dictionary of Music (1986) defines as 'a gradual crescendo and decrescendo on a sustained [sung] note', is known as one of the most difficult exercises for singers. Various experts consider this skill the ultimate test for a singer, indicating that it takes from months to several years of diligent and careful practice before a singer can expect to master the exercise.

The 'messa di voce' exercise is frequently associated with the 'candle test', although it remains unclear which historical personality first recommended this link. The 'candle test' is a centuries-old method used by singers to evaluate airflow. It simply consists of singing a vowel with a candle flame placed about five inches from the mouth. Depending on the
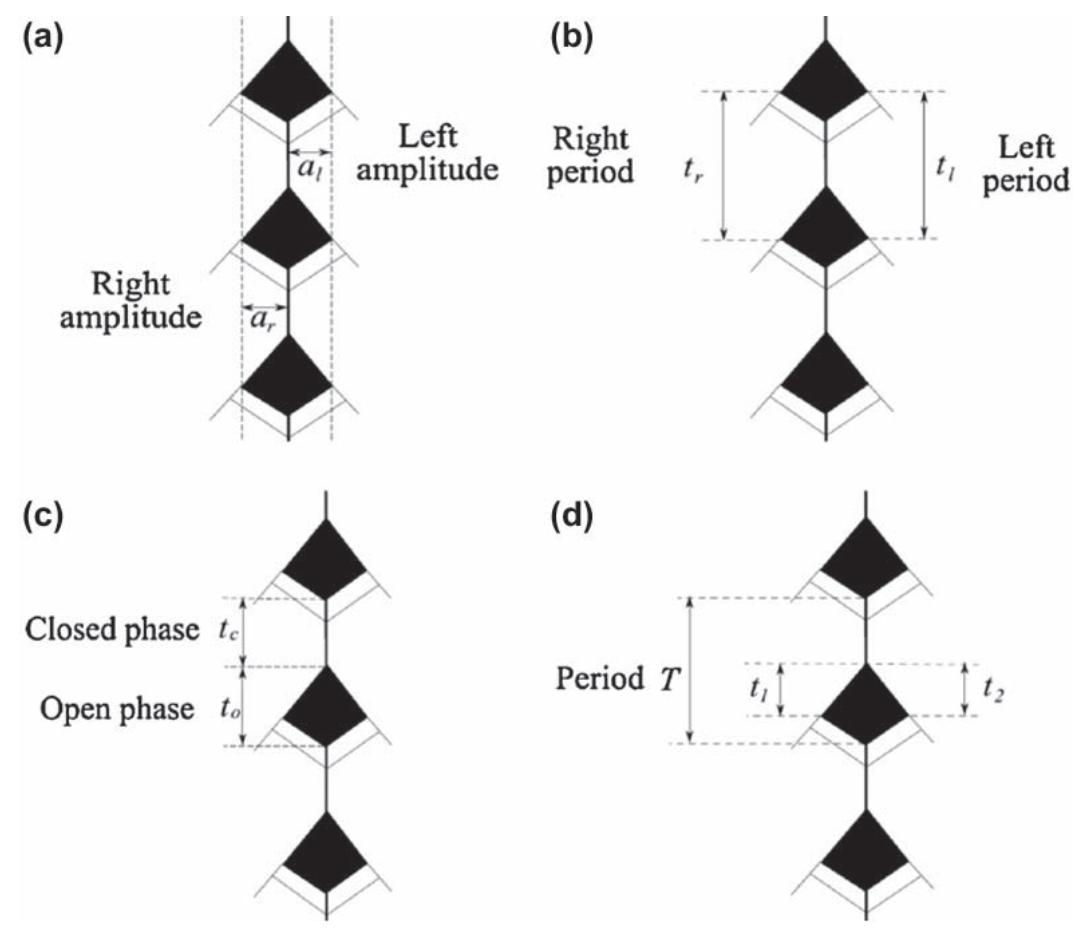

Figure 1. Schematic view of the physiological parameters of vocal fold vibrations, as shown by a videokymogram (from Manfredi et al. 2012, with permission) (5). 
exhaled airflow, the flame will waver very little (or not at all) or flutter wildly (7). During a 'messa di voce' by a trained singer, the flow increase at the $f f$ must remain minimal.

\section{'Messa di voce': the underlying physiology}

From a physiological point of view, a progressive increase in voice intensity - thus primarily in subglottic pressure-has several critical requirements $(8,9)$ :

1. constant adjustment of tension in the intrinsic laryngeal muscles, particularly the $\mathrm{m}$. vocalis and the m. cricothyroideus, in order to keep the fundamental frequency $(\mathrm{Fo})$ constant and to compensate for the enhanced passive strain in the folds, induced by the averaged higher vibration amplitude;

2. specific mechanical regulation at the transition from a 'one-mass-model' vibration in $p p$ (falsettolike) to a 'two-mass-model' (usual modal register), particularly at the early beginning of the crescendo;

3. permanent control of the voice quality in order to avoid audible noise (breathiness) appearance due to increase of the transglottic airflow, concomitantly with the increase of the lung pressure.

Actually, the performer must succeed in obtaining, at each intensity level, an exact balance between expiratory pressure and glottal impedance. Ideally, it results in only a slight increase in transglottic flow (10), which prevents blowing out the candle.

Hence the different acoustic and biomechanical parameters involved are: 1) subglottic pressure; 2) fundamental frequency (Fo, or 1/period); 3) sound pressure level (SPL); 4) glottal volume velocity; 5) vibration amplitude of each vocal fold for each cycle; 6) respective duration of the open and closed phases of each cycle; and 7) respective opening and closing speeds of each vocal fold.

In healthy larynges (e.g. of singing students), no significant asymmetries (in amplitude, frequency, and speed) are to be expected, but this is obviously not the case in pathology. The same is of course true for period and phase irregularities.

\section{Materials and methods}

\section{Kymography system}

The single line scanning system used in these experiments comprises a Lambert CCD-Kymocam with technical characteristics corresponding to the abovereported description, a rigid $90^{\circ}$ Wolf laryngeal tele- scope, a JVC-magnetoscope, and a monitor. The telescope has a magnifying facility, with narrow depth of field and critical sharpness adjustment.

\section{Vocal material}

A trained vocalist (baritone), non-professional singer, was asked to produce series of 'messa di voce' utterances on different pitches, avoiding vibrato. Vocal fold vibrations were recorded using the VKG system, and the subject could have a real-time visual control on the screen. After some preliminary trials, the subject became able to handle the scope himself and to find the optimal placement, combining comfort and quality of vocal fold image. During four different sessions, 62 recordings were achieved. The sound was also recorded by a Sennheiser microphone at $10 \mathrm{~cm}$ from the lips, for analysis of SPL and Fo (using PRAAT 5.3.10, 2012, by P. Boersma \& D. Weenink; www.praat.org). One of the recordings, considered as representative by the subject and providing a complete visualization of the endolarynx, was selected. For demonstration, 40 static pictures (single line scans), each showing two vibratory cycles, were taken at $125 \mathrm{~ms}$ intervals in order to cover the complete utterance $(5 \mathrm{~s})$. These images were enlarged and printed for manual measurements of the following parameters, as schematically shown in Figure 1: right and left period, right and left amplitude, open and closed phase, and right and left opening time.

All measurements were made manually, independently by two observers, and averaged.

The closed quotient is the quotient 'duration of closed phase/duration of cycle'.

An adequate calibration was achieved with respect to time and distance. For time, Fo was exactly known from PRAAT and period measurement on the microphone signal. For distance, scale paper was filmed without changing the focusing and with critical attention to maximal sharpness. This way of proceeding is inspired by Fex et al. (11) and has an inherent error of $5 \%-10 \%$.

\section{Program for automatic analysis}

First attempts to analyse automatically single line scans of vocal fold vibration were achieved by Qiu et al. (12). This approach is based on monodimensional active contours, where each line has its own energy to be minimized separately from other lines. With the VKG-Analyser used in the current experiments, a planar active contour is applied, i.e. the set of all points (two for each VKG line) is considered as a pair of lines (corresponding to left and right vocal folds) that contain a surface. During 
subsequent iterations, the contour is modified in order to adapt to the shape given by the dark pixels of the image. Planar snakes allow the algorithm to find a global minimum of the energy, thus performing a global optimization instead of a series of local ones. Moreover, in the VKG-Analyser, specific parameters are evaluated for the case of incomplete vocal fold closure.

A detailed description of the VKG-Analyser has been provided in a recent publication (5), and an earlier version of the VKG-Analyser has been reported in Manfredi et al. (2).

Each image in the sequence can be processed using a digital image-processing algorithm developed and optimized for the analysis of VKG recordings. It performs intensity adjustment, noise removal, and robust techniques for vocal fold edge detection to avoid fluctuations of the grey levels in regions at a distance from the vocal folds.

The vocal folds contour detection algorithm consists of two main steps: the first one defines an initial contour of the glottal area opening, using an adaptive threshold. A refining iterative procedure, based on active contours, is applied to the region, giving the final segmentation. The control parameters which drive both steps are determined automatically by the program. However, the user can manually adjust some of the controls for improving segmentation using a set of controls present in the user interface, as shown in Figure 2. As can be observed in the figure, the software allows individual selection of the desired frame(s) to be processed from the video recording. The central part of the window shows the selected frame, with the outcome of the edge detection algorithm (in red, open folds; in blue, fold edges not detected, closed).

Once the final contour has been obtained, the parameters of interest are evaluated. The software is designed so as to give a value of each parameter for each video frame, by averaging the parameters over all the vibration periods which can be observed in the frame. This reduces the variability of the results, by smoothing out noise, and eases the management of data, by giving a fixed number of values for a given video sequence, independently from the acquisition.

The program includes a user-friendly interface for importing the video sequences into the subject database and performing the analysis. It also allows storage and retrieval of the subject's data, display of tracked parameters, results, and statistics.

In the clinical field, the program has already been successfully applied to a set of normal subjects, and to patients with benign lesions of the vocal folds, such as polyps and cysts (4). For this research, the VKG-Analyser has been specifically adapted in order to save the required parameters that are evaluated for each frame (for instance, left amplitude and right amplitude, in addition to amplitude ratio).

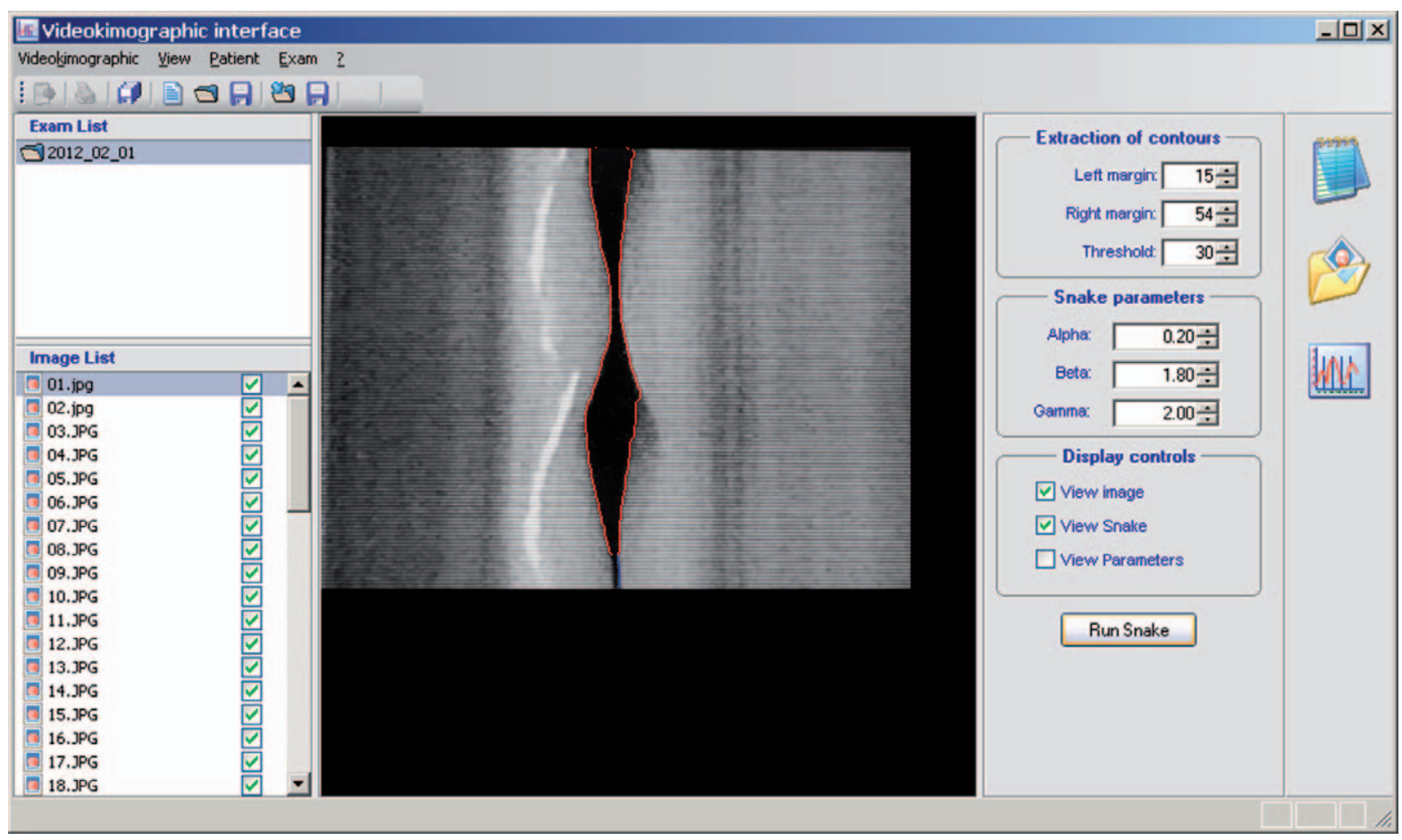

Figure 2.Videokymographic user interface. The central part of the window shows the selected frame, with the outcome of the edge detection algorithm (in red, open folds; in blue, fold edges not detected, closed). Time runs from top to bottom. The first closed phase is seen at the bottom of the picture. 


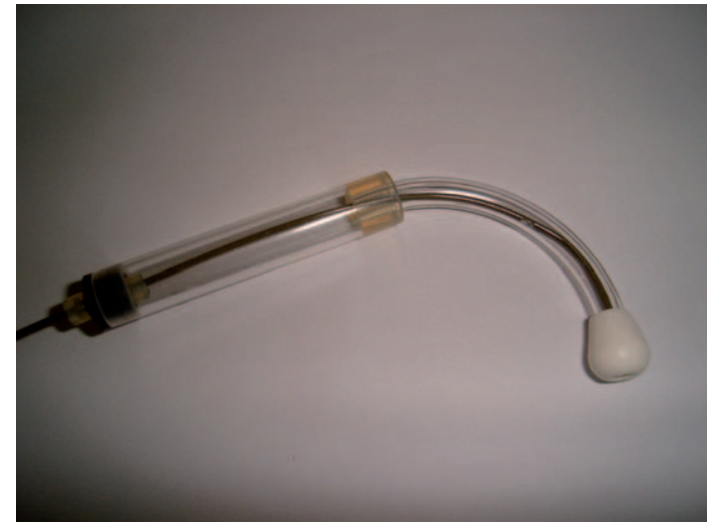

Figure 3. Self-designed device for indirect measures of subglottic pressure, using the short flow interruption method. The intra-oral pressure is measured with a Millar MikroTip catheter imbedded into the device.

For the purpose of this study, the vocalist-once trained in optimizing his 'messa di voce'-also repeated the exercise for separate indirect measurement of subglottic pressure and flow glottography. Ideally flow glottography could be combined with the single line scanning, but this is hard to achieve in a pedagogical situation. For indirect measures of subglottic pressure, the short flow interruption method was used (13). The intra-oral pressure was measured with a Millar MikroTip catheter imbedded in a self-designed device (Figure 3). Hertegard at al. (13) found that the indirect measures of subglottic pressure, obtained using the short flow interruption method, were strongly correlated with the direct measurements achieved by tracheal puncture. Of course, repeated voluntary interruptions of the phonation by lip closure - which are indispensable for the indirect measure of the subglottic pressureperturb to some extent the motor control of the 'messa di voce' exercise. This means that the display of the estimated subglottic has here only a value as documentation: it illustrates the main physiological substratum of the intensity changes.

The glottal volume velocity waveform was recorded with a Rothenberg mask and the MSIF2 inverse filtering system of Glottal Enterprises, Inc.

\section{Results}

Results are presented in Figures 4 to 14 .

Figure 4 gives a general impression of the microphone signal and the basic acoustic characteristics of a typical 'messa di voce' that will be analysed in the following graphs. Amplitude increases and decreases $(\sim 35 \mathrm{~dB}$ at $10 \mathrm{~cm})$ while Fo remains stable $(165 \mathrm{~Hz})$. Duration of the sequence is about $5 \mathrm{~s}$.

Figures 5, 6, and 7 show three characteristic kymograms, respectively, at the early beginning of the 'messa di voce' (there is a very short contact between the vocal edges), at approximately the middle part of the SPL rising phase of the 'messa di voce' (the closed phase is about one-fourth of the period), and at approximately the maximal SPL of the 'messa di voce' (the closed phase near half of the period).

Figure 8 gives an overview of changes in per cent over time in three important physiological parameters: amplitude of vibration (right + left), duration of the closed phase, and closed/open quotient (quotient of duration of the closed phase/duration of open phase). For clarity, an example was chosen in which
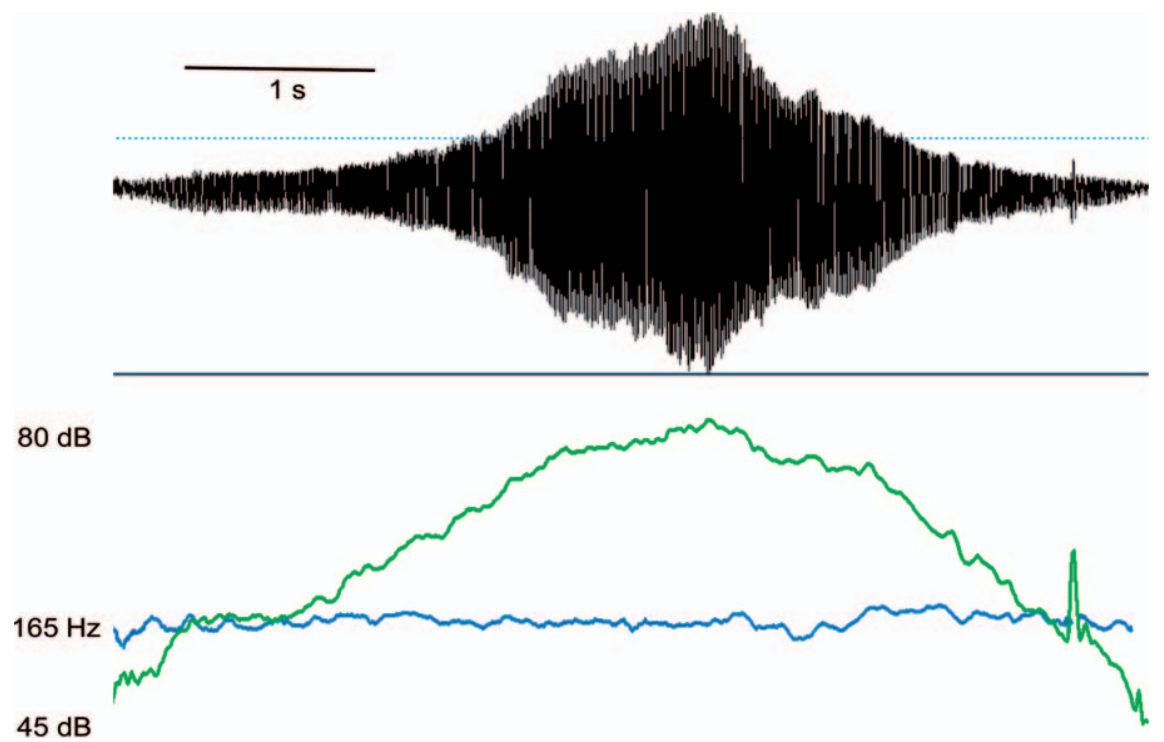

Figure 4. Microphone signal of the typical the 'messa di voce' analysed in the next figures, with plots of Fo and sound pressure level (at $10 \mathrm{~cm}$ from lips). 


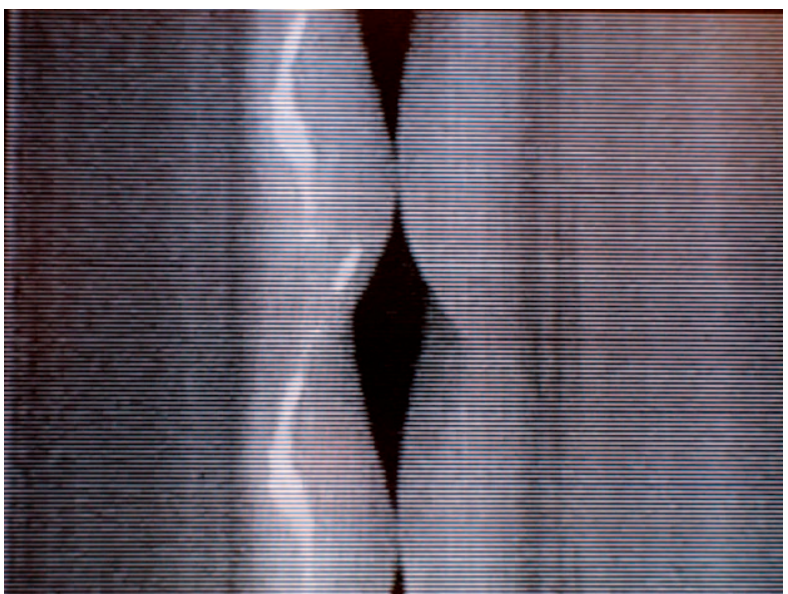

Figure 5. Videokymogram at the early beginning of the 'messa di voce': there is a very short contact between both vocal edges.

a vocal fold contact is already present at the beginning of the 'messa di voce'. Lack of glottic closure is of course also detected by the program. In the present example, the lowest value for each parameter is set as $100 \%$, in order to illustrate the relative changes. All these data result from hand-made measurements. The smoothing curves (least squares fit) are shown as solid lines.

Respective durations of both components of the open phase (i.e. the opening and the closing component) are compared over time in Figure 9; in Figure 10 , speed of opening and speed of closing of the vocal folds are plotted comparatively over time (all handmade measurements).

The data generated by the program for automatic analysis are compared with the data obtained by hand-made measurements in Figures 11 and 12. The correlation coefficients are 0.88 for the closed quotient and 0.84 for the amplitude.

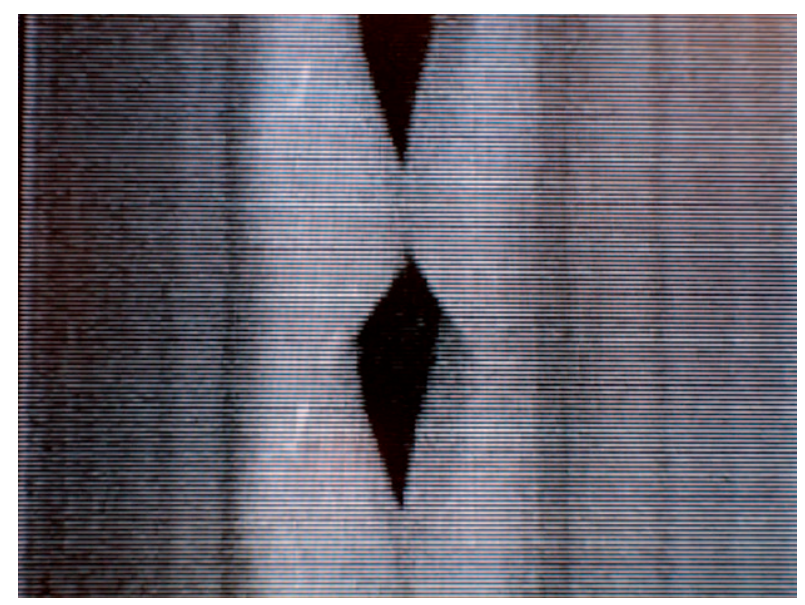

Figure 6. Videokymogram at approximately the middle of the SPL rising phase of the 'messa di voce': the closed phase is about one-fourth part of the period.

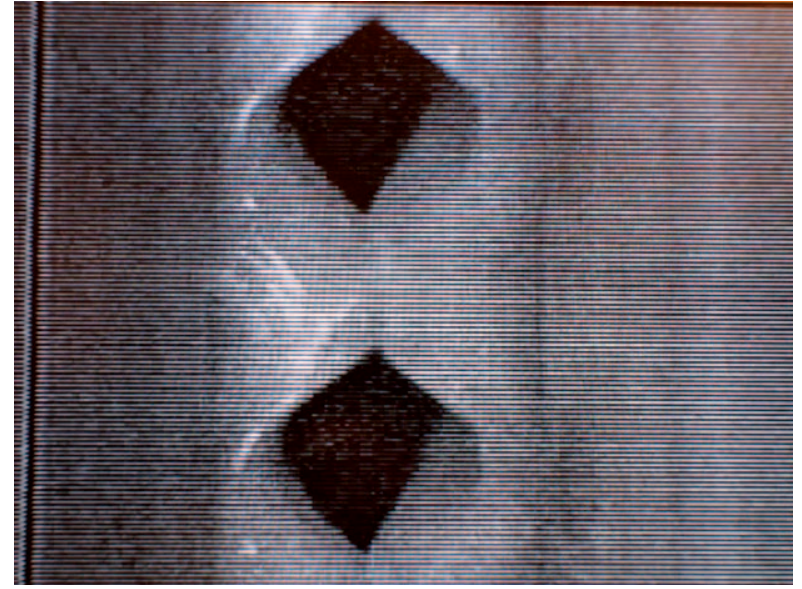

Figure 7. Videokymogram at approximately the maximal SPL of the 'messa di voce': the closed phase is near to the half of the period.

Results of the additional instrumental measurements are shown in Figures 13 and 14: As an estimate for subglottic pressure, the short flow interruption method provides values varying between about 3 and $20 \mathrm{~cm} \mathrm{H}_{2} \mathrm{O}(\mathrm{hPa})$ (Figure 13).

Figure 14 gives the glottal volume velocity waveform recorded with a flow glottograph (Rothenberg mask and inverse filtering system), showing a satisfactory control of the air flow around approximately $200-250 \mathrm{~mL} / \mathrm{s}$.

\section{Discussion}

\section{Global feedback on the acoustic phenomenon}

During the execution of the 'messa di voce' the changes of intensity are, in this vocalist, clearly correlated with the changes of breath pressure (13): this can be seen in Figure 13, although changes in spectral balance (singer's formant) may contribute to perception of increased loudness: Collyer et al. (14) found (in 'messa di voce') a linear correlation between sound pressure level (SPL) and spectral balance (ratio of power in $0-2 \mathrm{kHz}$ band to that in $2-4 \mathrm{kHz})$.

Ideally, one could expect the exercise to be performed as a symmetric triangle: a linear increase in loudness, followed by a linear decrease. A reasonable assumption, as suggested by Titze et al., is that in aiming at a linear crescendo and decrescendo, singers attempt to control the loudness of their voice linearly (15). However, the pedagogical assumptions of linearity and symmetry have received little critical assessment (14). These authors found that, even in trained classical singers, the change in SPL was frequently non-linear, and the shape of the SPL traces differed greatly between crescendo and decrescendo. 


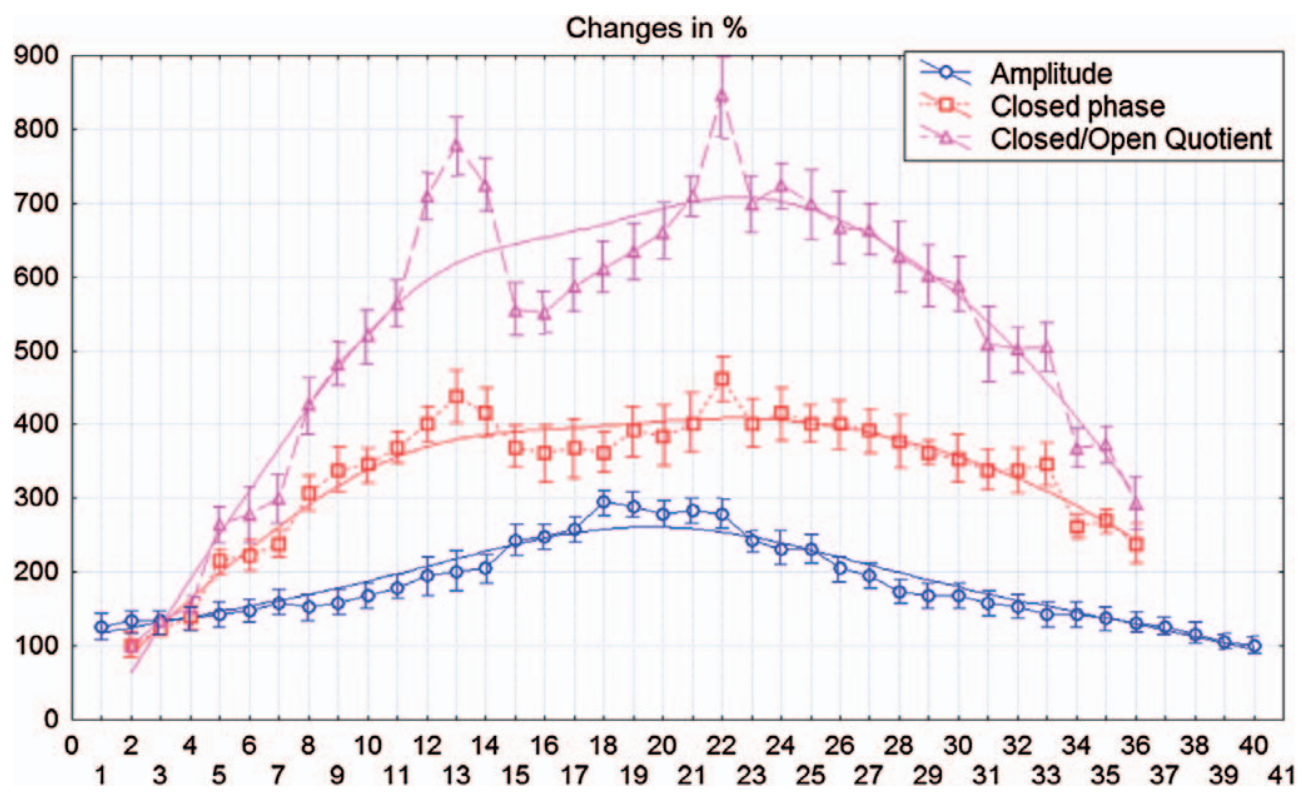

Figure 8. Comparative plots of changes in per cent across the 'messa di voce' of amplitude of glottal opening, duration of closed phase, and closed/open quotient (40 pictures at intervals of $125 \mathrm{~ms}$ ). The lowest value for each parameter is standardized to $100 \%$ (hand-made measurements with average and error bars).

The study of Cabrera et al. (16) points out that improving linearity is a difficult task for students. This suggests that feedback could be useful. A global acoustic overview of the process, provided as shortterm feedback, seems relevant to help the singing student (or performer) in enhancing linearity and symmetry. In the recording analysed here, and obtained at the end of a training session, the symmetry as well as the control of glottal resistance had considerably improved when compared with the first trials. The subject proved to be able to improve these parameters very rapidly when he received feedback.

\section{Detailed feedback on vocal fold biomechanics}

The cricothyroid and thyroarytenoid muscles are primarily involved in regulating the biomechanical properties of vocal fold vibration. As the vocal

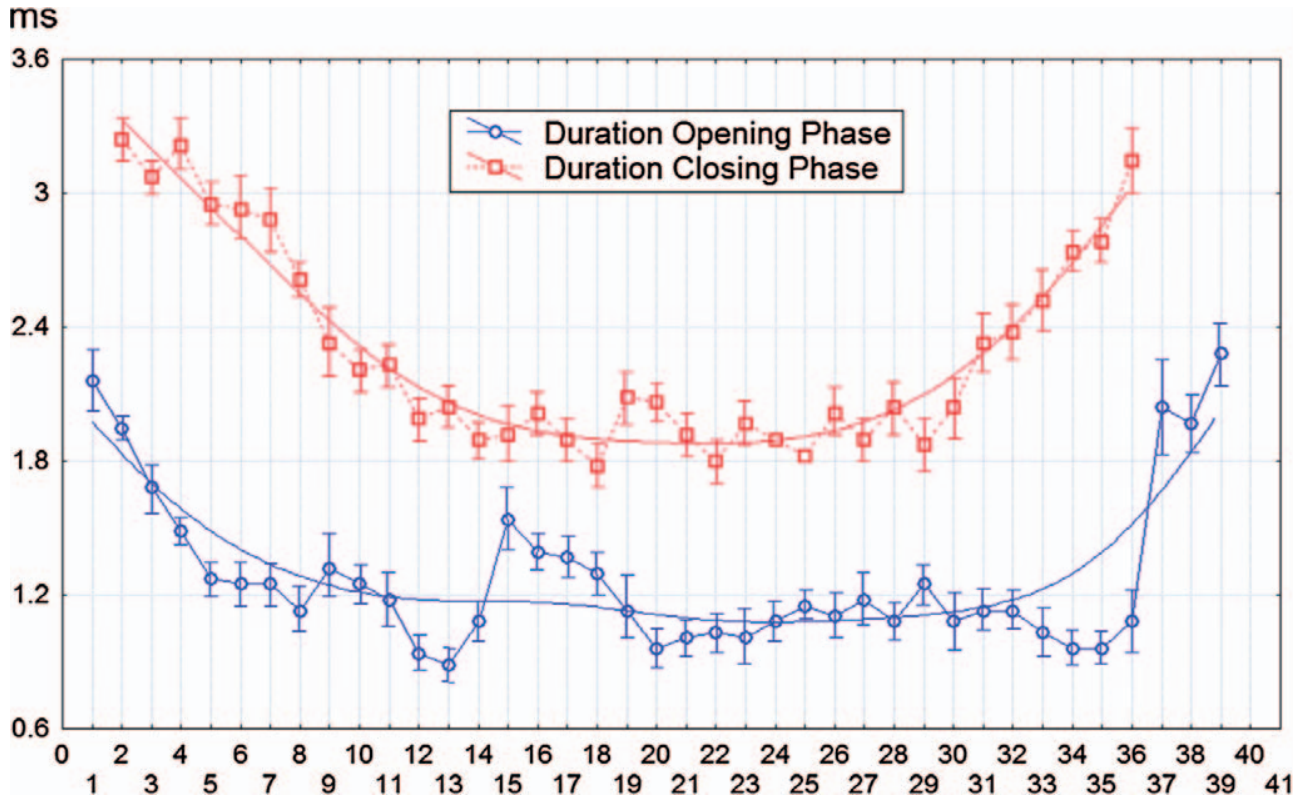

Figure 9. Comparative plots of duration of the opening phase and duration of the closing phase of the vocal folds across the 'messa di voce' (40 pictures at intervals of $125 \mathrm{~ms}$ ) (hand-made measurements with average and error bars). 


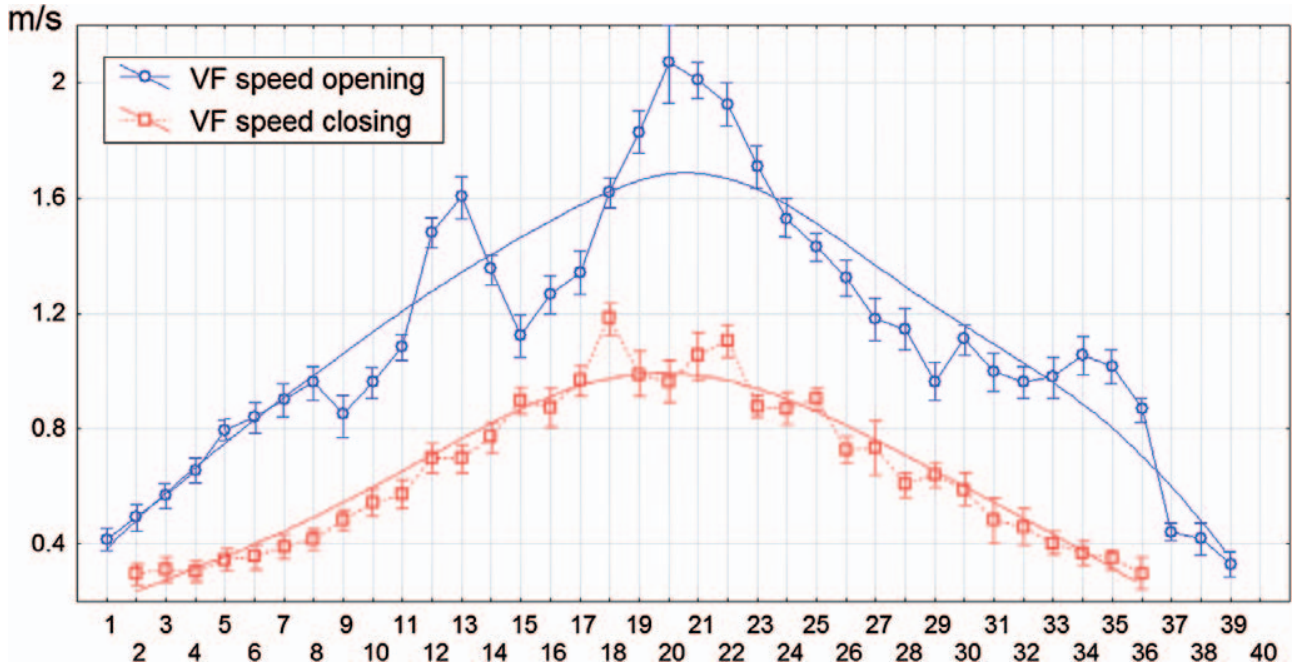

Figure 10. Comparative plots of the changes in opening speed and closing speed of the vocal folds across the 'messa di voce' (40 pictures at intervals of $125 \mathrm{~ms}$ ) (hand-made measurements with average and error bars).

intensity increases, it is expected that their tension will also be increased at a gradual pace to resist the growing breath pressure, but without compromising tonal accuracy. Conversely, when attempting a decrease in intensity, both muscles need to release their tension in response to reducing lung pressure. Furthermore, according to Titze (9) the singer usually needs to start the 'messa di voce' with a 'light' mechanism (near falsetto, with a vibration limited to the thin edge of the vocal fold, known as 'one-mass' model vibration), and then (preferably without break) to make a transition to the 'heavy mechanism' ('twomass' model).
Hence, the physiological mechanism of the 'messa di voce' seems to consist basically in an increase/ decrease of glottal impedance in parallel with the increase/decrease of the SPL, the SPL itself being closely related to the lung pressure. Videostroboscopy of the vocal fold vibration has demonstrated that the degree of glottal closure increases with intensity of phonation in normal subjects (17). Furthermore, also in normal subjects, the glottal impedance is well reflected by the electroglottographic closed quotient, and the quotient clearly increases with voice SPL; this increase in vocal fold closure is plausibly related to an active thickening of the vocal fold edge (in modal

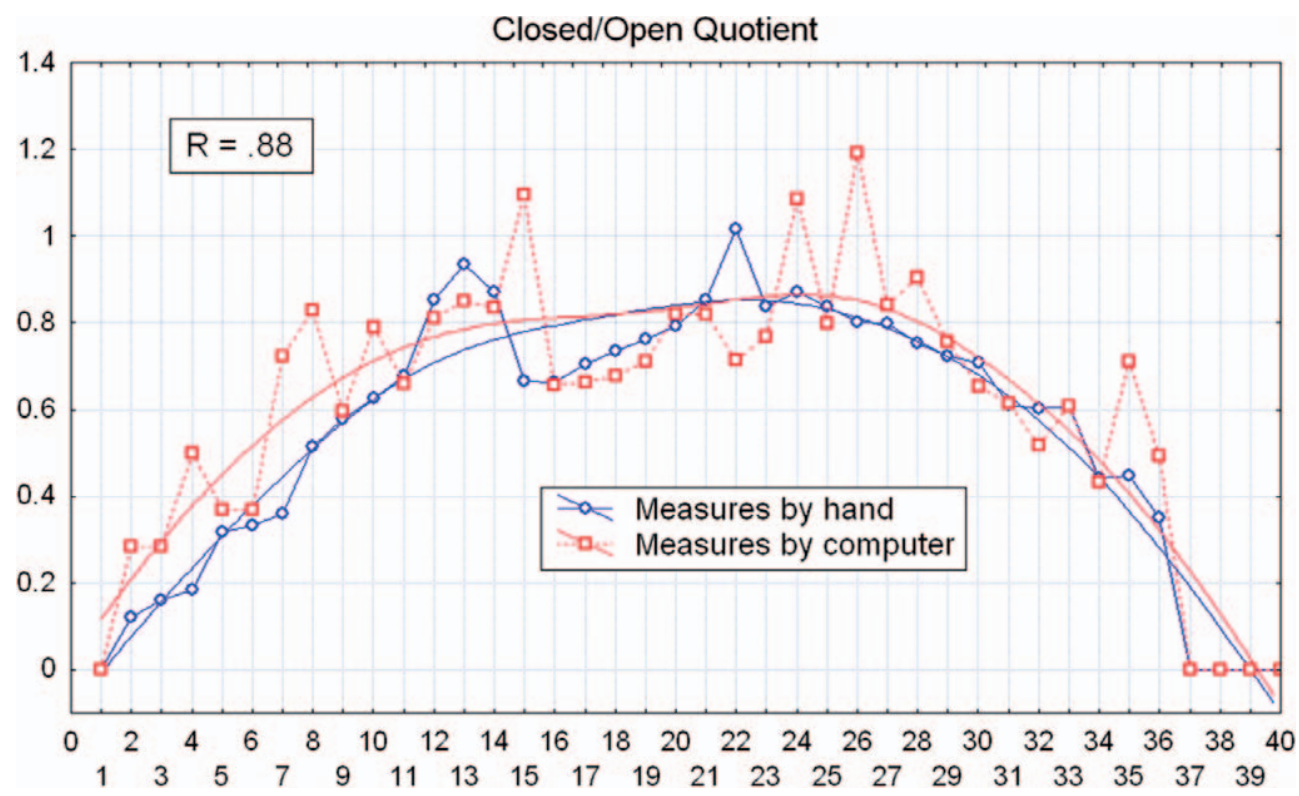

Figure 11. Comparative plots of the closed/open quotient as measured 'by hand' (average of two raters) and by the automatic analysis program. 


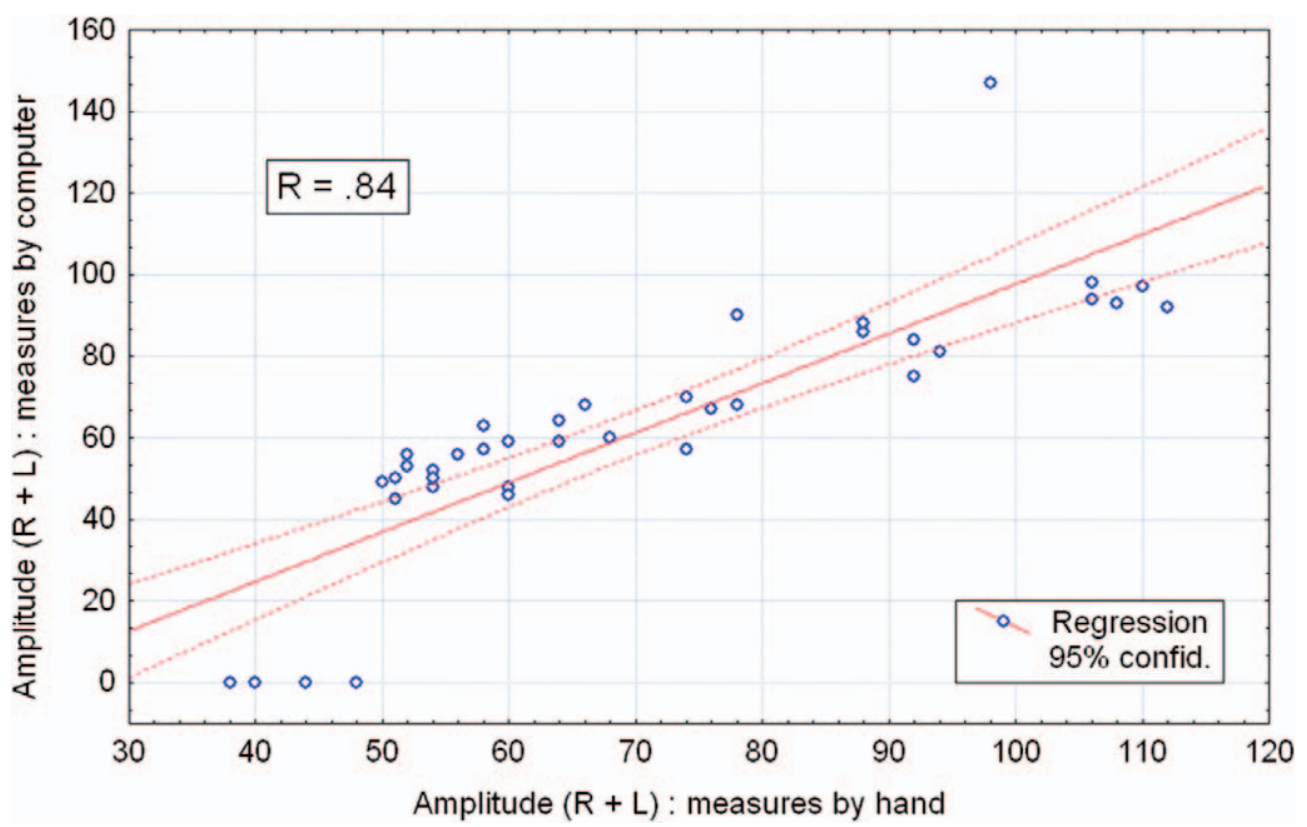

Figure 12. Correlation between the maximal amplitude of glottal opening (excursion of right vocal fold + excursion of left vocal fold) as measured 'by hand' (average of two raters) and by the automatic analysis program.

register) and to a longer closed phase of the vibratory cycle (18). This may account for the limited concomitant increase in transglottal flow (10). In the same way, Henrich et al. (19) found, in singers achieving crescendos-decrescendos, a negative correlation between open quotient and vocal intensity. Titze (8) has hypothesized (using computer modelling) that the maximal glottal source power should occur somewhere around a closed/open quotient of 0.5 , i.e. when open and closed phases are approximately equal (as observed in the demonstrated case; Figure 7). However, finding this kind of optimal adjustment may not be an easy task for every singer.

All these physiological concepts are reflected to various degrees in the quantitative analysis of the

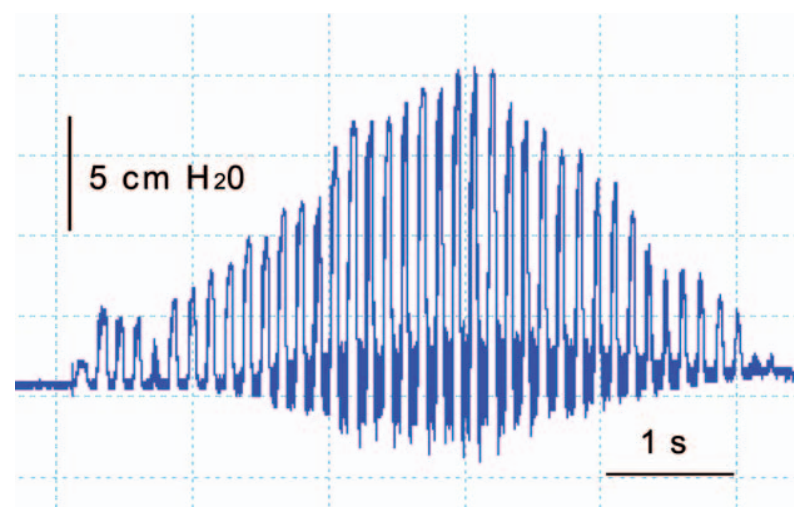

Figure 13. Plot of estimated subglottic pressure-as measured by the flow interruption method-across the 'messa di voce'. The 'thickening' of the baseline results from the superimposition of the interrupted microphone signal. kymographic recordings. For example, Figure 8 demonstrates that during the 'messa di voce' the main relative increase/decrease of closed/open quotient $(100 \%-700 \%)$ greatly exceeds that of amplitude of vibration (less than $100 \%-300 \%$ ). Figure 9 shows that the durations of the opening and closing phases evolve in parallel, but that the closing phases always greatly exceed the opening phases; conversely (Figure 10), the vocal folds systematically open faster than they close.

Of course this is only a single example out of a large number of recordings obtained from one single vocalist, but there is little doubt that the singer and the singing pedagogue can benefit from this kind of feedback information. In this experiment,

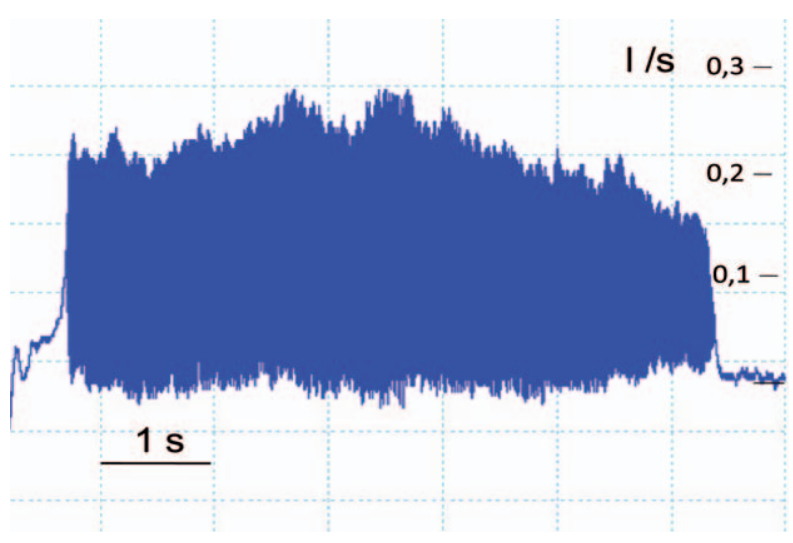

Figure 14. Plot of the amplitude of the flow glottogram (glottal volume velocity waveform obtained by the inverse filtering method) across the 'messa di voce'. 
the subject was-after a short time training and some direct visual feedback - perfectly able to handle the endoscope himself, finding the optimal position that caused minimal discomfort and required no tongue protrusion. In future, paediatric transnasal fiberscopes will probably improve these aspects still further.

\section{The need for an automatic analysis program}

However, these quantitative data are of practical value only if they are available immediately after the 'messa di voce'. Manual computation is so timeconsuming that it makes the method unsuitable for practical pedagogical applications. Therefore, in this work, a new user-friendly tool, the VKG-Analyser $(2,5)$, is used and tried out for the automatic extraction, tracking, and computing of quantitative parameters from the VKG images. The software tool has already been successfully tested for reliability on a set of synthetic and real VKG images and tried out in a clinical context $(4,5)$. In the present study, comparative plots of the closed/open quotient measured manually (average of two raters) and by the automatic analysis program confirm the efficacy of the analysis program: the correlation coefficient between the two measurements is 0.88 (Figure 12). For amplitude measurements, the correlation coefficient is 0.84 (Figure 13). In practice, this is sufficient for training and educational purposes. In this study and for a valid comparison, the computer measurements were made on the same pictures that were used for the hand-made measurements, but the automatic computations can be directly achieved on the video recording in quasi-real time (5). In that case, all cycle pictures are analysed.

Further advantages of the program are a zooming feature, allowing a more precise evaluation of the proper threshold in enlarged images, and of its plasticity (specific parameters or quotients). Actually the VKG-Analyser can be applied either to pathologic subjects or to singers, as the extracted parameters are the same for both cases. The interesting features for a 'messa di voce' in a trained singer are obviously different from those for the investigation of pathological vocal folds. Perturbation and asymmetry will be most relevant in pathology, while in singing one will rather focus - as shown in the current study-on relative amplitude and specific quotients (closed/ opening/closing).

\section{Additional measurements}

Non-invasive additional measurements, such as estimated subglottic pressure and flow-glottography, may meaningfully contribute to the analysis, to the understanding, and to the feedback of the 'messa di voce'. Both of them are fitted for real-time visual feedback: the direct visualization of the pressure data will help in increasing symmetry (Figure 13), while monitoring the phonation flow plays the role of the historical candle in front of the mouth (Figure 14).

More generally, short-term relevant visual information about the acoustic result and the directly related physical and physiological parameters would hopefully help the singing teacher to make his comments and advice on the student's performances clearer and more understandable. With a limited amount of training, singers - who are known to have an excellent sensory-motor control of their vocal tract - can handle the scope themselves, following in this the historical example of Manuel Garcia.

As the results presented here concern a first trial on a single subject; they only demonstrate that this novel application is feasible. The actual pedagogical interest remains to be proven. Further studies could, for example, compare a group of singing students trained with feedback and a group trained in the 'traditional' way, or the effect of the feedback method in students unable to reach a satisfactory result with the 'traditional' approach.

\section{Conclusion}

'Single line scanning' of the vocal fold vibrations appears to be-beside its clinical usefulness-also suitable for investigating a specific technical voice exercise (and musical ornament) such as the 'messa di voce'. It makes possible a precise quantification over time of the essential physiological parameters characterizing the changes of glottal impedance concomitant with the progressive increase and decrease of the lung pressure. A condition is that the subject can tolerate the presence of the endoscope while performing the exercise.

However, introduction of a valid and userfriendly automatic analysis program of kymography images appears indispensable for opening such new applications in the field of voice pedagogics by short-term feedback. The data provided by the automatic analysis program show a strong correlation with hand-made measurements. Additional measurements, such as subglottic pressure and instantaneous phonation flow, can meaningfully be correlated with the data obtained from the kymography images.

Declaration of interest: The authors report no conflicts of interest. 


\section{References}

1. Svec J, Schutte HK. Videokymography: high-speed line scanning of vocal fold vibration. J Voice. 1996;10:201-5.

2. Manfredi C, Bocchi L, Bianchi S, Migali N, Cantarella G. Objective vocal fold vibration assessment from videokymographic images. Biomed Signal Process Control. 2006;1: 129-36.

3. Svec J, Sram F, Schutte HK. Videokymography in voice disorders: what to look for? Ann Otol Rhinol Laryngol. 2007;116:172-80.

4. Piazza C, Mangili S, Del Bon F, Gritti F, Manfredi C, Nicolai P, et al. Quantitative analysis of videokymography in normal and pathological vocal folds: a preliminary study. Eur Arch Otorhinolaryngol. 2012;269:207-12.

5. Manfredi C, Bocchi L, Cantarella G, Peretti G. Videokymographic image processing: objective parameters and user-friendly interface. Biomed Signal Process Control. 2012;7:192-201.

6. Qiu Q, Schutte HK. New generation videokymography for routine clinical vocal fold examination. Laryngoscope. 2006;116:1824-8.

7. Taylor DC. A rational method of voice culture based on a scientific analysis of all systems, ancient and modern. New York: MacMillan; 1922.

8. Titze IR. Principles of voice production. Englewood Cliffs, NJ: Prentice Hall; 1994.

9. Titze IR. More on messa di voce. Journal of Singing. 1996;52:31-2.

10. Smitheran JR, Hixon TJ. A clinical method for estimating laryngeal airway resistance during vowel production. J Speech Hear Disord. 1981;46:138-46.
11. Fex S, Fex B, Hirano M. A clinical procedure for linear measurement at the vocal fold level. J Voice. 1991;5: 328-31.

12. Qiu Q, Schutte HK, Gu L, Yu Q. An automatic method to quantify the vibration properties of human vocal folds via videokymography. Folia Phoniatr Logop. 2003;55: 128-36.

13. Hertegard S, Gauffin J, Lindestadt PA. A comparison of subglottal and intraoral pressure measurements during phonation. J Voice. 1995;9:149-55.

14. Collyer S, Davis PJ, Thorpe CW, Callaghan J. Sound pressure level and spectral balance linearity and symmetry in the messa di voce of female classical singers. J Acoust Soc Am. 2007;121:1728-36.

15. Titze IR, Long R, Shirley GI, Stathopoulos E, Ramig LO, Carroll LM, et al. Messa di voce: an investigation of the symmetry of crescendo and decrescendo in a singing exercise. J Acoust Soc Am. 1999;105:2933-40.

16. Cabrera D, Yadav M, Kenny DT. Linearity in the loudness envelope of the messa di voce. Proceedings of ACOUSTICS, 2-4 November 2011, Gold Coast, Australia, 5 pp.

17. Sodersten M, Lindestadt PA. Glottal closure and perceived breathiness during phonation in normally speaking subjects. J Speech Hear Res. 1990;33:601-11.

18. Dejonckere PH. Control of fundamental frequency and glottal impedance with increasing sound pressure in normal and pathological voices. Voice. 1994;3:10-16.

19. Henrich N, d'Alessandro C, Doval B. Glottal open quotient in singing: measurements and correlation with laryngeal mechanisms, vocal intensity and fundamental frequency. J Acoust Soc Am. 2005;117:1417-30. 